\title{
Sonographic Diagnosis of an Acute Lateral Meniscus Tear in a Division I Collegiate American Football Player
}

\author{
Olubusola A. Brimmo, MD ${ }^{1}$ Patrick A. Smith, MD ${ }^{1}$ Cristi R. Cook, DVM, MS ${ }^{2}$ James L. Cook, DVM, PhD ${ }^{1}$ \\ ${ }^{1}$ Department of Orthopaedic Surgery, University of Missouri, \\ Columbia, Missouri \\ ${ }^{2}$ Comparative Orthopaedic Laboratory, University of Missouri, \\ Columbia, Missouri \\ Address for correspondence James L. Cook, DVM, PhD, Missouri \\ Orthopaedic Institute, University of Missouri, 1100 Virginia Ave., \\ DC953.00 Columbia, MO 65212 (e-mail: cookjl@health.missouri.edu).
}

J Knee Surg Rep 2015;1:57-59.

\begin{abstract}
Keywords

- meniscal tears

- magnetic resonance imaging

- ultrasonography

- diagnosis

Preoperative diagnosis of meniscal tears in the United States is typically determined from magnetic resonance imaging (MRI). However, MRI diagnosis of meniscal pathology is not a point-of-injury imaging modality. This results in potential treatment delays. Ultrasonography provides a method for highly reliable, immediate, and point-of-injury diagnosis of meniscal pathology. We present a case of an acute tear of the lateral meniscus in a collegiate athlete that was diagnosed in the training room immediately after injury using ultrasonography. The diagnosis was subsequently verified by MRI and arthroscopy. The patient was treated with partial meniscectomy and experienced an uneventful recovery with return to sport 3 weeks after injury. This case report supports the potential capabilities of ultrasonography as an effective and efficient point-of-injury diagnostic tool for athletes with suspected meniscal pathology.
\end{abstract}

Elite American football players are a high-risk population for meniscal injuries of the knee, with an incidence rate as high as $12.4 \%{ }^{1}$ An accurate preoperative diagnosis of meniscal pathology depends on diagnostic imaging of the affected joint, with magnetic resonance imaging (MRI) considered the current diagnostic method of choice in US collegiate athletes. ${ }^{2}$ Although MRI is considered the gold standard for diagnosing meniscal pathology, there are many limitations to its use such as expense, availability, patient size, claustrophobia, phonophobia, magnet-sensitive implants, motion artifacts, and imaging time. ${ }^{3}$ For athletes, the inability for MRI to serve as a point-of-injury diagnostic modality further limits its usefulness. Finally, MRI is associated with misdiagnosis of meniscal pathology in a significant number of cases. ${ }^{4}$

A recent study from Cook et $\mathrm{al}^{4}$ provided data regarding the diagnostic capabilities of ultrasonography for assessing meniscal abnormalities in patients with acute knee pain. In this study, ultrasonography was two times more likely than

received

November 4, 2014 accepted after revision

March 9, 2015

published online

May 26, 2015
MRI to correctly determine the presence or absence of meniscal pathology in this cohort of patients. The purpose of this case report is to describe the implementation of pointof-injury ultrasonography for immediate diagnosis of an acute tear of the lateral meniscus in a Division I collegiate American football player.

\section{Case History}

An 18-year-old collegiate American football player with no history of left knee pain presented in the training room with left lateral knee pain immediately after twisting his left knee participating in cone drills during practice. After twisting his knee, he tried to continue with practice, however lateral knee pain caused him to voluntarily discontinue and seek medical attention. Physical examination by the team physician revealed mild effusion of the left knee. He had lateral pain with hyperextension and flexion. There were no signs of ligamentous instability. He was tender over the posterolateral joint
Copyright $\odot 2015$ by Thieme Medical Publishers, Inc., 333 Seventh Avenue, New York, NY 10001, USA. Tel: +1(212) 584-4662.
License terms

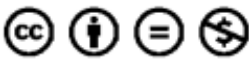

10.1055/s-0035-1551549. ISSN 2326-2729. 


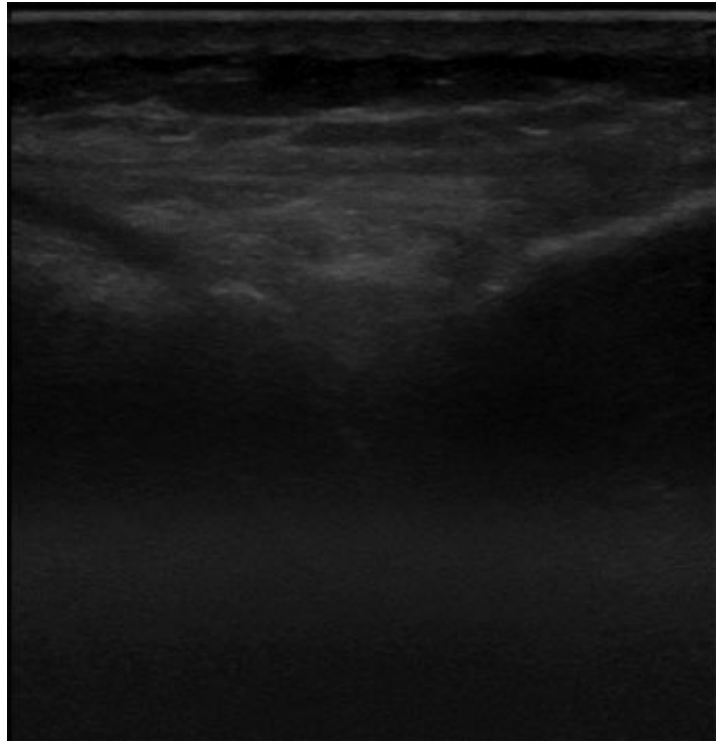

Fig. 1 Ultrasound scan of the left knee demonstrates a loss of uniform echogenicity of the central and posterior portions of lateral meniscus indicative of a lateral radial meniscus tear. The ultrasound was performed in the training room using a 10 to $14 \mathrm{MHz}$ linear transducer immediately after the injury occurred.

line and a McMurray test was positive for lateral pain, but with no "pop." Differential diagnoses included lateral meniscal tear, chondral injury or bone contusion, or posterolateral capsular sprain.

Ultrasonography of the knee was requested by the team physician and performed by a radiologist with expertise in musculoskeletal ultrasonography while the subject was in the training room. The ultrasonographic examination was performed using a 10 to $14 \mathrm{MHz}$ linear transducer (GE Medical Systems, Logiq i, Milwaukee, WI) and a standardized assessment system as previously described. ${ }^{4}$ Ultrasonographic findings included irregular shape, slight abaxial displacement, and loss of uniform echogenicity of the central and posterior portions of lateral meniscus with a small amount of subjacent hypoechoic fluid (-Fig. 1). No other ultrasonographic abnormalities were identified on assessment of the left knee. The ultrasonographic diagnosis was posterior-central lateral meniscal tear with associated effusion. On the basis of the ultrasonographic diagnosis, the patient was withheld from all athletic activities until further diagnostic imaging was performed.

On the basis of the presiding medical protocol at our institution, multiplanar, multisequence noncontrast MRI of the left knee was performed 2 days after his injury, which revealed a minimally displaced radial tear of the lateral meniscus and a small, ruptured Baker cyst. The patient underwent arthroscopic surgery on the left knee 3 days after injury, which confirmed an avascular, complex, white/white zone radial tear of the central-posterior portion of the lateral meniscus (-Fig. 2). The complex radial tear was determined to be unrepairable, and arthroscopic partial lateral meniscectomy was performed.

Postoperatively, he was started on isometric quadriceps exercises with early range of motion. He returned to noncontact football drills 15 days after surgery, and fully participated

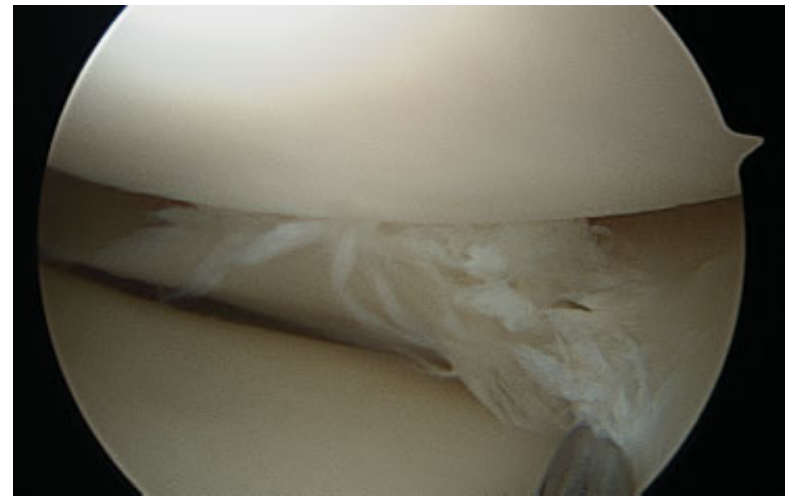

Fig. 2 Arthroscopic image demonstrating an avascular complex radial lateral meniscus tear within the left knee. Surgery was performed 3 days after the injury occurred.

in practice 18 days after surgery. He has not experienced any further difficulty with his knee. All patient confidentiality is protected in this report according to the US Health Insurance Portability and Accountability Act (HIPAA).

\section{Discussion}

In this case report, ultrasonographic imaging of the knee performed in the training room immediately after injury was effective in correctly diagnosing a complex, radial tear of the lateral meniscus in a collegiate American football player. Ultrasonographic assessment of the knee has been reported to have excellent capabilities for diagnosis of meniscal tears. ${ }^{4-9}$ Reported sensitivities for ultrasonographic diagnosis of meniscal tears range from 83.3 to $100 \%$ and reported specificities range from 69.2 to $95 \%{ }^{4-9}$ Differences in sensitivity and specificity among studies likely stem from variations in patient population, ultrasonographer's technique and level of expertise, and the reference standard used. In the study by Cook et al comparing ultrasonography and MRI for diagnosis of meniscal pathology in patients with acute knee pain, they reported a sensitivity of $91.2 \%$ and specificity of $84.2 \%$ for ultrasonography compared with a sensitivity of $91.7 \%$ and specificity of $66.7 \%$ for MRI using arthroscopic diagnosis as the reference standard. ${ }^{4}$ Perhaps more importantly, ultrasonography was associated with a correct classification rate of $89.5 \%$ compared with $81.1 \%$ for MRI, making ultrasonography two times more likely than MRI to correctly diagnose the presence or absence of meniscal pathology in our study.

Portability of current ultrasonographic equipment has created a platform for its use in evaluating primary injuries at point of injury. High-quality ultrasound machines are also available in laptop and handheld versions. A portable laptop ultrasound machine with high-resolution linear probes was used for imaging in this case, which provided an accurate diagnosis at the athletic facility. This methodology provides the potential for immediate decision-making after injury so as best to inform the medical staff and athletic personnel, and protect the athlete. It also provides for preoperative diagnosis of meniscal injuries for athletes who do not have access to MRI because of the financial or logistical limitations. However, it 
should be pointed out that ultrasonography has not been validated for diagnosis of pathology of the anterior cruciate ligament, posterior cruciate ligament, articular cartilage, or other structures within the knee. Therefore, MRI has advantages over ultrasonography for comprehensive diagnostic imaging of the injured knee.

Ultrasonographic examination of the knee using a standardized assessment system to determine the presence or absence of meniscal pathology provides the potential for immediate, point of injury medical decision-making regarding optimal management of injured athletes.

\section{References}

1 Brophy RH, Barnes R, Rodeo SA, Warren RF. Prevalence of musculoskeletal disorders at the NFL Combine-trends from 1987 to 2000. Med Sci Sports Exerc 2007;39(1):22-27

2 Oei EHG, Ginai AZ, Hunink MGM. MRI for traumatic knee injury: a review. Semin Ultrasound CT MR 2007;28(2):141-157
3 Crues JV III, Mink J, Levy TL, Lotysch M, Stoller DW. Meniscal tears of the knee: accuracy of MR imaging. Radiology 1987;164(2): 445-448

4 Cook JL, Cook CR, Stannard JP, et al. MRI versus ultrasonography to assess meniscal abnormalities in acute knees. J Knee Surg 2014; 27(4):319-324

5 Najafi J, Bagheri S, Lahiji FA. The value of sonography with micro convex probes in diagnosing meniscal tears compared with arthroscopy. J Ultrasound Med 2006;25(5):593-597

6 Park GY, Kim JM, Lee SM, Lee MY. The value of ultrasonography in the detection of meniscal tears diagnosed by magnetic resonance imaging. Am J Phys Med Rehabil 2008;87(1):14-20

7 Wareluk P, Szopinski KT. Value of modern sonography in the assessment of meniscal lesions. Eur J Radiol 2012;81(9): 2366-2369

8 Shetty AA, Tindall AJ, James KD, Relwani J, Fernando KW. Accuracy of hand-held ultrasound scanning in detecting meniscal tears. J Bone Joint Surg Br 2008;90(8):1045-1048

9 Alizadeh A, Babaei Jandaghi A, Keshavarz Zirak A, Karimi A, Mardani-Kivi M, Rajabzadeh A. Knee sonography as a diagnostic test for medial meniscal tears in young patients. Eur J Orthop Surg Traumatol 2013;23(8):927-931 